\title{
Evidence for anisotropy of cosmic acceleration ${ }^{\star}$
}

\author{
Jacques Colin ${ }^{1}$, Roya Mohayaee ${ }^{1}$, Mohamed Rameez ${ }^{2}$, and Subir Sarkar ${ }^{3}$ \\ 1 CNRS, UPMC, Institut d'Astrophysique de Paris, 98 bis Bld Arago, Paris, France \\ 2 Niels Bohr Institute, University of Copenhagen, Blegdamsvej 17, 2100 Copenhagen, Denmark \\ 3 Rudolf Peierls Centre for Theoretical Physics, University of Oxford, Parks Road, Oxford OX1 3PU, UK \\ e-mail: s.sarkar@physics.ox.ac.uk
}

Received 22 July 2019 / Accepted 18 October 2019

\begin{abstract}
Observations reveal a "bulk flow" in the local Universe which is faster and extends to much larger scales than are expected around a typical observer in the standard $\Lambda \mathrm{CDM}$ cosmology. This is expected to result in a scale-dependent dipolar modulation of the acceleration of the expansion rate inferred from observations of objects within the bulk flow. From a maximum-likelihood analysis of the Joint Light-curve Analysis catalogue of Type Ia supernovae, we find that the deceleration parameter, in addition to a small monopole, indeed has a much bigger dipole component aligned with the cosmic microwave background dipole, which falls exponentially with redshift $z: q_{0}=q_{\mathrm{m}}+\boldsymbol{q}_{\mathrm{d}} \cdot \hat{n} \exp (-z / S)$. The best fit to data yields $q_{\mathrm{d}}=-8.03$ and $S=0.0262(\Rightarrow d \sim 100 \mathrm{Mpc})$, rejecting isotropy $\left(q_{\mathrm{d}}=0\right)$ with $3.9 \sigma$ statistical significance, while $q_{\mathrm{m}}=-0.157$ and consistent with no acceleration $\left(q_{\mathrm{m}}=0\right)$ at $1.4 \sigma$. Thus the cosmic acceleration deduced from supernovae may be an artefact of our being non-Copernican observers, rather than evidence for a dominant component of "dark energy" in the Universe.
\end{abstract}

Key words. cosmology: observations - dark energy - large-scale structure of Universe

\section{Introduction}

The foundations of the current standard model of cosmology date back nearly a century to when essentially no data were available. In particular the Universe was assumed to be exactly isotropic and homogeneous, with space-time described by the maximally symmetric Friedmann-Lemaître-RobertsonWalker metric, and occupied by ideal fluids with purely diagonal energy-momentum tensors (Peebles 1994). Subsequently it has been recognised that the distribution of galaxies, which is a biased tracer of the underlying distribution of the dominant dark matter, is rather inhomogeneous. Counts-in-spheres of galaxy catalogues have suggested that there is a transition to (statistical) homogeneity on scales exceeding $\sim 100 \mathrm{Mpc}$ (Hogg et al. 2005; Scrimgeour et al. 2012), although sufficiently large volumes have not yet been surveyed to establish this definitively. This is however the expectation in the current standard cosmological model if the observed large-scale structure has grown under gravity in the sea of dark matter, starting with an initially Gaussian random field of small density perturbations with an approximately scale-invariant spectrum. Detailed observations of the temperature fluctuations in the cosmic microwave background (CMB) have broadly confirmed this model (Planck Collaboration I 2019). However several anomalies have been noted such as the lack of correlations on large angular scales, the quadrupole-octupole alignment, and the hemispherical power asymmetry, which seem to imply a violation of statistical isotropy and scale-invariance of primordial

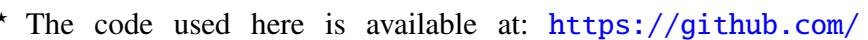
rameez3333/Dipole_JLA
}

perturbations; nevertheless there is no consensus yet on either their physical nature or their origin (Schwarz et al. 2016).

In our real Universe there are "peculiar motions" due to the local inhomogeneity and anisotropy of surrounding structure. These are non-negligible, for example our Local Group of galaxies moves with respect to the universal expansion at $620 \pm$ $15 \sim \mathrm{km} \mathrm{s}^{-1}$ towards $\ell=271.9 \pm 2^{\circ}, b=29.6 \pm 1.4^{\circ}$, as is inferred from the observed dipolar modulation of the CMB temperature (Kogut et al. 1993; Planck Collaboration I 2019). Moreover diverse observations reaching out as far as $\sim 300 \mathrm{Mpc}$, for example, by Lauer \& Postman (1994), Hudson et al. (2004), Watkins et al.(2009), Lavaux et al. (2010), Feldman et al. (2010), Colin et al. (2011), Feindt et al. (2013), and Magoulas et al. (2016), have not seen the expected $\sim 1 / r$ fall-off of the peculiar velocity in the standard $\Lambda$ cold dark matter (CDM) cosmology. The odds of this happening by chance in that framework can be estimated by querying Hubble volume simulations of largescale structure formation, for example, Dark Sky (Skillman et al. 2014). Less than $1 \%$ of Milky Way-like observers should observe the bulk flow $\left(>250 \mathrm{~km} \mathrm{~s}^{-1}\right.$ extending to $\left.z>0.03\right)$ that we observe (Rameez et al. 2018). Thus we are not comoving observers but are "tilted" relative to the idealised Hubble flow (Tsagas 2010). The implications of this have been discussed for measurements of the Hubble parameter $H_{0}$ (Hess \& Kitaura 2014), but not for the inference of cosmic acceleration.

Since cosmological observables are formulated in the "CMB frame" in which the Universe is supposedly perfectly isotropic, it is in any case always necessary to correct what we measure from our relative moving frame. For example the observed redshifts of the Type Ia supernovae (SNe Ia) in catalogues like Joint Light-curve Analysis (JLA; Betoule et al. 2014) 
have been corrected to convert from the heliocentric frame to the CMB frame. The methodology used follows earlier work (Conley et al. 2011) which used bulk flow observations made back in 2004 (Hudson et al. 2004) and moreover assumed that there is convergence to the CMB frame beyond $150 \mathrm{Mpc}$. Since this is not in accordance with subsequent deeper observations, we use only the heliocentric redshifts and reverse the corrections applied to the magnitudes to examine whether the deceleration parameter measured in our (bulk flow) rest frame can indeed differ from that of comoving observers in the model universe (Tsagas 2010). Such theoretical considerations imply (Tsagas 2011; Tsagas \& Kadiltzoglou 2015) that there should be a dipole asymmetry in the derived cosmic deceleration parameter $q_{0}$ towards the bulk flow direction. In this work we find a significant $(3.9 \sigma)$ indication of such a dipole, and also that the monopole in $q_{0}$ decreases simultaneously in significance (to $1.4 \sigma$ ). Hence not only is the indication for acceleration statistically marginal (Nielsen et al. 2016), it probably arises because we are tilted observers located in a bulk flow, rather than due to the effect of a cosmological constant or dark energy.

\section{Joint Light-curve Analysis}

We used the most up-to-date publicly available sample of supernova light-curve properties and directions: the SDSS-II/SNLS3 JLA catalogue (Betoule et al. 2014). This consists of $740 \mathrm{spec}-$ troscopically confirmed SNe Ia, including several low redshift $(z<0.1)$ samples, three seasons of SDSS-II $(0.05<z<0.4)$, and three years of SNLS $(0.2<z<1)$ data; these samples are all calibrated consistently in the Spectral Adaptive Light-curve Template 2 (SALT2) scheme. This assigns three parameters to each supernova: the apparent magnitude $m_{B}^{*}$ at maximum in the rest-frame $B$ band and the light-curve shape and colour corrections, $x_{1}$ and $c$. The distance modulus is then given by

$\mu_{\mathrm{SN}}=m_{B}^{*}-M+\alpha x_{1}-\beta c$,

where $\alpha$ and $\beta$ are assumed to be constants, as is $M$ the absolute $\mathrm{SNe}$ Ia magnitude, as befits a standard candle. In the standard $\Lambda C D M$ cosmological model this is related to the luminosity distance $d_{\mathrm{L}}$ as

$$
\begin{aligned}
\mu & \equiv 25+5 \log _{10}\left(d_{\mathrm{L}} / \mathrm{Mpc}\right), \quad \text { where, } \\
d_{\mathrm{L}} & =(1+z) \frac{d_{\mathrm{H}}}{\sqrt{\Omega_{k}}} \sin \left(\sqrt{\Omega_{k}} \int_{0}^{z} \frac{H_{0} \mathrm{~d} z^{\prime}}{H\left(z^{\prime}\right)}\right), \text { for } \Omega_{k}>0 \\
& =(1+z) d_{\mathrm{H}} \int_{0}^{z} \frac{H_{0} \mathrm{~d} z^{\prime}}{H\left(z^{\prime}\right)}, \text { for } \Omega_{k}=0 \\
& =(1+z) \frac{d_{\mathrm{H}}}{\sqrt{\Omega_{k}}} \sinh \left(\sqrt{\Omega_{k}} \int_{0}^{z} \frac{H_{0} \mathrm{~d} z^{\prime}}{H\left(z^{\prime}\right)}\right), \text { for } \Omega_{k}<0 \\
d_{\mathrm{H}} & =c / H_{0}, \quad H_{0} \equiv 100 h \mathrm{~km} \mathrm{~s}^{-1} \mathrm{Mpc}^{-1}, \\
H & =H_{0} \sqrt{\Omega_{\mathrm{M}}(1+z)^{3}+\Omega_{k}(1+z)^{2}+\Omega_{\Lambda}} .
\end{aligned}
$$

In this equation, $d_{\mathrm{H}}$ is the Hubble distance and $H$ the Hubble parameter ( $H_{0}$ being its present value), and $\Omega_{\mathrm{M}}, \Omega_{\Lambda}, \Omega_{k}$ are the matter, cosmological constant, and curvature densities in units of critical density. In the $\Lambda \mathrm{CDM}$ model these are related by the cosmic sum rule $1=\Omega_{\mathrm{M}}+\Omega_{\Lambda}+\Omega_{k}$. However we make no such model assumptions and simply expand the luminosity distance $d_{\mathrm{L}}$ in a Taylor series to examine its second derivative, i.e. the acceleration (see Sect. 3). This is because acceleration is a kinematic quantity and can be measured without making any assumptions about the dynamics underlying the universal expansion. There may be concern that such a Taylor expansion fails at

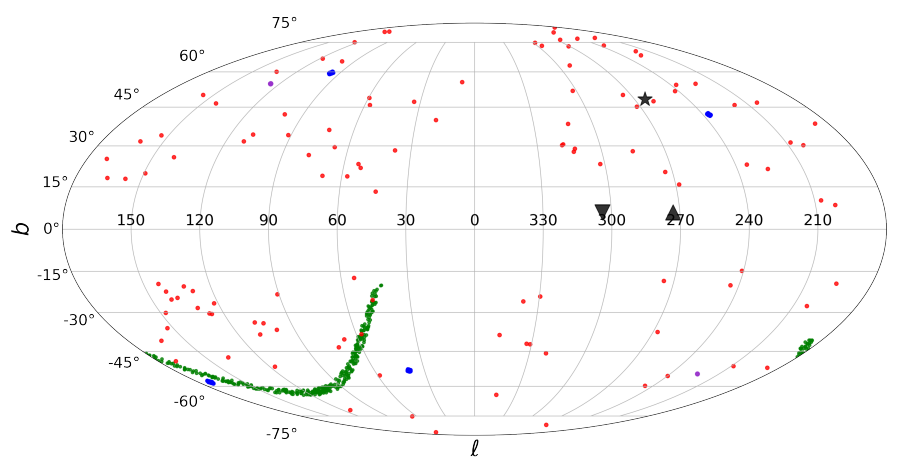

Fig. 1. Sky distribution of the 4 sub-samples of the JLA catalogue in galactic coordinates: SDSS (red dots), SNLS (blue dots), low redshift (green dots), and HST (black dots). The 4 big blue dots show clusters of many individual SNe Ia. The directions of the CMB dipole (star), the SMAC bulk flow (triangle) and the $2 \mathrm{M}++$ bulk flow (inverted triangle) are also shown.

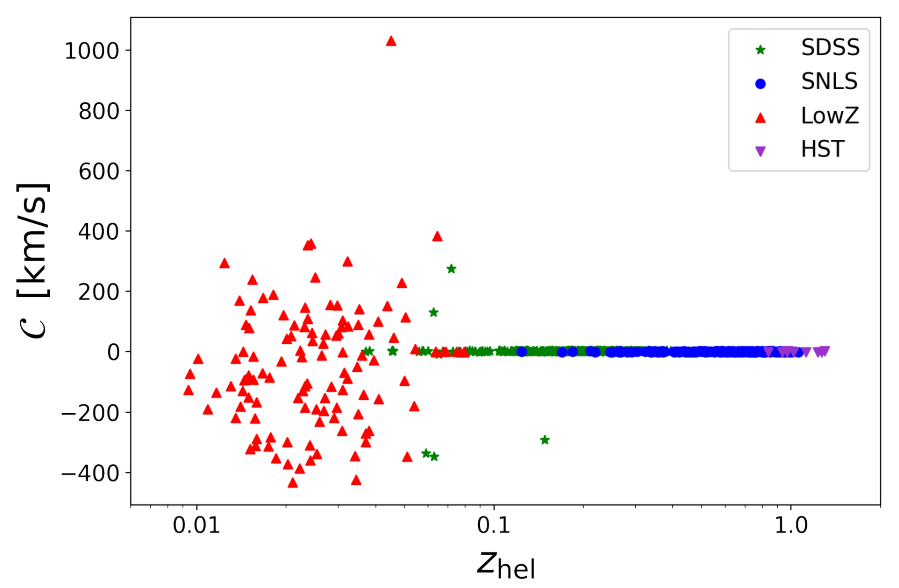

Fig. 2. Peculiar velocity corrections applied to the JLA catalogue. The velocity parameter $C$ in Eq. (3) is shown vs. the observed redshift.

high redshift, however we verified that $d_{\mathrm{L}}$ in the best-fit $\Lambda \mathrm{CDM}$ model differs by only $7 \%$ even at $z=1.3$ (the highest redshift in the JLA sample), which is much less than the measurement uncertainty. Indeed the Taylor expansion fits the data just as well as $\Lambda \mathrm{CDM}$.

Figure 1 is a Mollewide projection of the directions of the 740 $\mathrm{SNe}$ Ia in galactic coordinates. As a consequence of the diverse survey strategies of the sub-samples that make up the JLA catalogue, its sky coverage is patchy and anisotropic. While the low redshift objects are spread out unevenly across the sky, the intermediate redshift objects from SDSS are mainly confined to a narrow disc at low declination, while the high redshift objects from SNLS are clustered along four specific directions.

The JLA analysis (Betoule et al. 2014) corrects the observed redshifts in the heliocentric frame, $z_{\text {hel }}$, in order to obtain the cosmological redshifts, $z_{\mathrm{CMB}}$, after accounting for peculiar motions in the local Universe. These corrections are carried over unchanged from an earlier analysis (Conley et al. 2011), which in turn cites an earlier method (Neill et al. 2007) and the peculiar velocity model of Hudson et al. (2004). It is stated that the inclusion of these corrections allow SNe Ia with redshifts down to 0.01 to be included in the cosmological analysis, in contrast to earlier analyses (Riess et al. 2007) which employed only SNe Ia down to $z=0.023$.

In Fig. 2 we scrutinise these corrections by exhibiting the velocity parameter $C$, defined as 
$C=\left[\left(1+z_{\text {hel }}\right)-\left(1+z_{\mathrm{CMB}}\right)\left(1+z_{\mathrm{d}}\right)\right] \times c$,

where $z_{\text {hel }}$ and $z_{\mathrm{CMB}}$ are as tabulated by JLA, while $z_{\mathrm{d}}$ is given by (Davis et al. 2011)

$z_{\mathrm{d}}=\sqrt{\frac{1-\boldsymbol{v}_{\mathrm{CMB}-\odot \cdot \hat{n} / c}}{1+\boldsymbol{v}_{\mathrm{CMB}-\odot \cdot \hat{n} / c}}}-1$,

where $\boldsymbol{v}_{\mathrm{CMB}-\odot}$ is $369 \mathrm{~km} \mathrm{~s}^{-1}$ in the direction of the CMB dipole (Kogut et al. 1993) and $\hat{n}$ is the unit vector in the direction of the supernova. It can be seen in Fig. 2 that SNe Ia beyond $z \sim 0.06$ have been assumed to be stationary w.r.t. the CMB rest frame, and corrections applied only to those at lower redshifts. It is not clear how these corrections were made beyond $z \sim 0.04$, which is the maximum extent to which the Streaming Motions of Abell Clusters (SMAC) sample (Hudson et al. 2004) extends. This has a bulk velocity of $687 \pm 203 \mathrm{~km} \mathrm{~s}^{-1}$ towards $\ell=260 \pm 13^{\circ}, b=0 \pm 11^{\circ}$ out to $z=0.04$ at $90 \%$ C.L., and a bulk velocity of $372 \pm 127 \mathrm{~km} \mathrm{~s}^{-1}$ towards $\ell=273^{\circ}, b=6^{\circ}$ generated by sources beyond $200 h^{-1} \mathrm{Mpc}(\Rightarrow z \simeq 0.064)$ at $98 \%$ C.L. If the peculiar velocity field is not discontinuous, the SNe Ia immediately outside this volume should have comparable velocities. Figure 2 indicates however that the JLA peculiar velocity corrections have arbitrarily assumed that the bulk flow abruptly disappears at this point. The JLA analysis (Betoule et al. 2014) allows SNe Ia beyond this distance to only have an uncorrelated velocity dispersion of $c \sigma_{z}=150 \mathrm{~km} \mathrm{~s}^{-1}$. In the absence of any evidence of convergence to the CMB rest frame, this assumption is unjustified since it is very possible that the observed bulk flow stretches out to much larger scales. There have been persistent claims of a "dark flow" extending out to several hundreds of megaparsec (Kashlinsky et al. 2009, 2010, 2011), although this is still under debate. At any rate the value of $c \sigma_{z}$ should be determined by fitting to the data, rather than put in by hand.

At this point it is worth noting the anisotropy of the JLA catalogue. Out of the $740 \mathrm{SNe}$ Ia, 551 are in the hemisphere pointing away from the CMB dipole. With respect to the $372 \pm 127 \mathrm{~km} \mathrm{~s}^{-1}$ bulk flow of the model (Hudson et al. 2004) from which the redshifts of the local SNe Ia have been corrected, only 108 are in the upper hemisphere while 632 are in the lower hemisphere. With respect to the direction of the abnormally high flow reported by $6 \mathrm{dFGSv}$, the largest and most homogeneous peculiar velocity sample of nearly 9000 galaxies (Magoulas et al. 2016), 103 SNe Ia are in the upper hemisphere while 637 are in the lower hemisphere.

The subsequent Pantheon catalogue (Scolnic et al. 2018), which incorporates 308 additional SNe Ia (many from the PanSTARRS survey), continues to suffer from these problems. While the flow model (Carrick et al. 2015) from which the redshifts of the Pantheon sample have been corrected go out to $z \sim 0.067$, this model has a residual bulk flow of $159 \pm 23 \mathrm{~km} \mathrm{~s}^{-1}$ attributed to sources beyond $z=0.067$, and 890 of the 1048 Pantheon $\mathrm{SNe}$ are in the hemisphere opposite to the direction of this flow.

Both JLA and Pantheon include SNe to which anomalously large peculiar velocity corrections have been applied at redshifts far higher than the limit to which the corresponding flow models extend. Two of the many such examples are SDSS2308 in JLA at $z=0.14$ (the outlier in Fig. 2) and SN2246 in Pantheon at $z=0.194$.

We used the heliocentric redshifts tabulated by JLA (Betoule et al. 2014) and subtracted out the bias corrections applied to $m_{B}^{*}$. For the Pantheon catalogue (Scolnic et al. 2018) the $z_{\text {hel }}$ values and individual contributions to the covariance are not public, and moreover there are unresolved concerns about the accuracy of the data therein (Rameez 2019) so we cannot use it.

\section{Cosmological analysis}

We now compare the distance modulus (Eq. (1)) obtained from the JLA sample with the apparent magnitude (Eq. (2)) using the maximum likelihood estimator (MLE; Nielsen et al. 2016). Since we wish to analyse the data without making assumptions about the matter content or the dynamics, we use the kinematic Taylor series expansion of the luminosity distance up to the third term (Visser 2004) as follows:

$d_{\mathrm{L}}\left(z_{\mathrm{hel}}\right)=\frac{c z_{\text {hel }}}{H_{0}}\left\{1+\frac{1}{2}\left[1-q_{0}\right] z_{\text {hel }}-\frac{1}{6}\left[1-q_{0}-3 q_{0}^{2}+j_{0}+\frac{k c^{2}}{H_{0}^{2} a_{0}^{2}}\right] z_{\text {hel }}^{2}\right\}$,

where $q \equiv-\ddot{a} a / \dot{a}^{2}$ is the cosmic deceleration parameter in the CMB frame, defined in terms of the scale factor of the universe $a$ and its derivatives w.r.t. proper time, $j=\dot{a} / a H^{3}$ is the cosmic "jerk", and $-k c^{2} /\left(H_{0}^{2} a_{0}^{2}\right)$ is just $\Omega_{k}$. We note that the last two appear together in the coefficient of the $z^{3}$ term so these cannot be determined separately. In the $\Lambda \mathrm{CDM}$ model, $q_{0} \equiv \Omega_{\mathrm{M}} / 2-\Omega_{\Lambda}$.

To look for a dipole in the deceleration parameter, we allow it to have a direction dependence written as

$q=q_{\mathrm{m}}+\boldsymbol{q}_{\mathrm{d}} \cdot \hat{n} \mathcal{F}(z, S)$,

where $q_{\mathrm{m}}$ and $q_{\mathrm{d}}$ are the monopole and dipole components, while $\hat{n}$ is the direction of the dipole and $\mathcal{F}(z, S)$ describes its scale dependence. We consider four representative functional forms as follows:

(a) Constant: $\mathcal{F}(z, S)=1$ independent of $z$,

(b) Top hat: $\mathcal{F}(z, S)=1$ for $z<S$, and 0 otherwise,

(c) Linear: $\mathcal{F}(z, S)=1-z / S$, and

(d) Exponential: $\mathcal{F}(z, S)=\exp (-z / S)$.

Owing to the anisotropic sky coverage of the dataset, it would be hard to find $\hat{n}$ from the data, so we choose it to be along the CMB dipole direction. This is reasonable as the directions of the reported bulk flows (Hudson et al. 2004; Watkins et al. 2009; Lavaux et al. 2010; Feldman et al. 2010; Colin et al. 2011; Feindt et al. 2013; Magoulas et al. 2016) are all within $\sim 40^{\circ}$ of each other and of the CMB dipole. Later we allow the direction to vary to demonstrate that our result is indeed robust.

We maximise a likelihood constructed earlier (March et al. 2011; Nielsen et al. 2016), simultaneously with respect to the four cosmological parameters $q_{\mathrm{m}}, j_{0}-\Omega_{k}, q_{\mathrm{d}}$, and $S$, as well as the eight parameters that go into the standardisation of the SNe Ia candles: $\alpha, \beta, M_{0}, \sigma_{M_{0}}, x_{1,0}, \sigma_{x_{1,0}}, c_{0}$, and $\sigma_{c_{0}}$. While our analysis is frequentist, it is equivalent to the Bayesian hierarchical model of March et al. (2011), which indeed yielded the same result (Shariff et al. 2016) as the frequentist analysis of Nielsen et al. (2016) when applied to the JLA catalogue.

In Table 1 we show how this compares with using the prevalent constrained $\chi^{2}$ method used by, for example Betoule et al. (2014), wherein an arbitrary error $\sigma_{\text {int }}$ is added to each data point and varied until a good fit (with $\chi^{2}=1 /$ d.o.f.) is obtained to the assumed model. This may be appropriate for parameter estimation, but not for model selection.

However as seen in Table 2 the quality of fit improves further ( $-2 \log \mathcal{L}_{\text {max }}$ decreases) when $q_{0}$ is allowed to have a dipole. In the best fit where this has an exponentially decaying form $\propto \mathrm{e}^{-z / S}$, the dipole $q_{\mathrm{d}}=-8.03$ is much larger than the monopole $q_{\mathrm{m}}=-0.157$ 
Table 1. Tilted local universe, with $\sigma_{z}$ set to zero, fitted to data with the constrained $\chi^{2}$ method.

\begin{tabular}{lcccccccc}
\hline \hline & $q_{\mathrm{m}}$ & $q_{\mathrm{d}}$ & $S$ & $j_{0}-\Omega_{k}$ & $\alpha$ & $\beta$ & $M_{0}$ & $\sigma_{\text {int }}$ \\
\hline Tilted universe & -0.268 & -6.54 & 0.0297 & -0.517 & 0.135 & 3.04 & -19.053 & 0.124 \\
No tilt $\left(q_{\mathrm{d}}=0\right)$ & -0.307 & 0 & - & -0.523 & 0.133 & 3.03 & -19.047 & 0.133 \\
\hline
\end{tabular}

Table 2. Tilted local universe, with $\sigma_{z}$ set to zero, fitted to data with the MLE.

\begin{tabular}{|c|c|c|c|c|c|c|c|c|c|c|c|c|c|}
\hline & $-2 \log \mathcal{L}_{\max }$ & $q_{\mathrm{m}}$ & $q_{\mathrm{d}}$ & $S$ & $j_{0}-\Omega_{k}$ & $\alpha$ & $x_{1,0}$ & $\sigma_{x_{1,0}}$ & $\beta$ & $c_{0}$ & $\sigma_{c_{0}}$ & $M_{0}$ & $\sigma_{M_{0}}$ \\
\hline Tilted universe & -208.28 & -0.157 & -8.03 & 0.0262 & -0.489 & 0.135 & 0.0394 & 0.931 & 3.00 & -0.0155 & 0.071 & -19.027 & 0.114 \\
\hline No tilt $\left(q_{\mathrm{d}}=0\right)$ & -189.52 & -0.166 & 0 & - & -0.460 & 0.133 & 0.0396 & 0.931 & 2.99 & -0.014 & 0.071 & -19.028 & 0.117 \\
\hline No accn. $\left(q_{\mathrm{m}}=0\right)$ & -205.98 & 0 & -6.84 & 0.0384 & -0.836 & 0.134 & 0.0365 & 0.931 & 2.99 & -0.014 & 0.071 & -19.002 & 0.115 \\
\hline
\end{tabular}

Notes. The BIC for the models above is $-129.00,-123.45$, and -133.31 , providing strong evidence for the last model.

Table 3. Tilted local universe, with $\sigma_{z}$ left floating, fitted to data with the MLE.

\begin{tabular}{|c|c|c|c|c|c|c|c|c|c|c|c|c|c|c|}
\hline & $-2 \log \mathcal{L}_{\max }$ & $q_{\mathrm{m}}$ & $q_{\mathrm{d}}$ & $S$ & $j_{0}-\Omega_{k}$ & $\alpha$ & $x_{1,0}$ & $\sigma_{x_{1,0}}$ & $\beta$ & $c_{0}$ & $\sigma_{c_{0}}$ & $M_{0}$ & $\sigma_{M_{0}}$ & $c \sigma_{z}\left[\mathrm{~km} \mathrm{~s}^{-1}\right]$ \\
\hline Tilted universe & -216.90 & -0.154 & -6.33 & 0.0305 & -0.497 & 0.134 & 0.0395 & 0.932 & 3.04 & -0.0158 & 0.071 & -19.022 & 0.106 & 241 \\
\hline No tilt $\left(q_{\mathrm{d}}=0\right)$ & -203.23 & -0.187 & 0 & - & -0.425 & 0.133 & 0.0398 & 0.932 & 3.05 & -0.0151 & 0.071 & -19.032 & 0.106 & 274 \\
\hline No accn. $\left(q_{\mathrm{m}}=0\right)$ & -214.74 & 0 & -5.60 & 0.0350 & -0.833 & 0.133 & 0.0368 & 0.932 & 3.04 & -0.0145 & 0.071 & -19.000 & 0.106 & 243 \\
\hline
\end{tabular}

Notes. The BIC for the models above is $-131.01,-130.55$, and -135.46 , providing positive evidence for the last model.

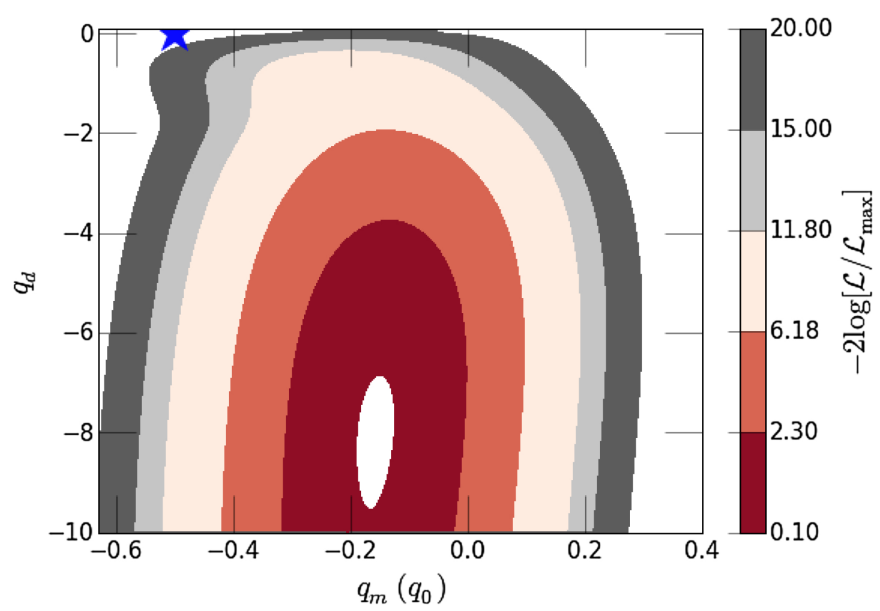

Fig. 3. Monopole and dipole components of the cosmological deceleration parameter (inferred from the JLA catalogue of $740 \mathrm{SNe}$ Ia). The 1,2 , and $3 \sigma$ contours (corresponding to $-2 \log \mathcal{L} / \mathcal{L}_{\max }=2.3,6.18$, and 11.8 , respectively) are shown, profiling over all other parameters. The vertical scale for the magnitude of the dipole is compressed by $\times 10$ relative to the horizontal scale for the monopole. The value of $q_{0}$ for the standard $\Lambda \mathrm{CDM}$ model is shown as a blue star.

and its scale parameter is $S=0.0262$, indicating that the impact of the bulk flow dominates over any isotropic acceleration out to $z \sim 0.1$. Since $\Delta_{\mathrm{BIC}}$ between the model with $q_{\mathrm{d}}=0$ and the model with $q_{\mathrm{m}}=0$ is 9.86 , this constitutes strong evidence against a universe that is accelerating isotropically. In the presence of this dipole, $q_{\mathrm{m}}=0$ is disfavoured at only $1.4 \sigma$. In other words, in a universe in which we have theoretical reasons to expect a dipolar modulation in the deceleration parameter in the direction of our motion through the CMB, there is no significant evidence for a non-zero value of its monopole component. Figure 4 shows the 1,2 , and $3 \sigma$ contours in the likelihood around the maximum as a function of $q_{\mathrm{d}}$ and $q_{\mathrm{m}}$, profiling over all other parameters.
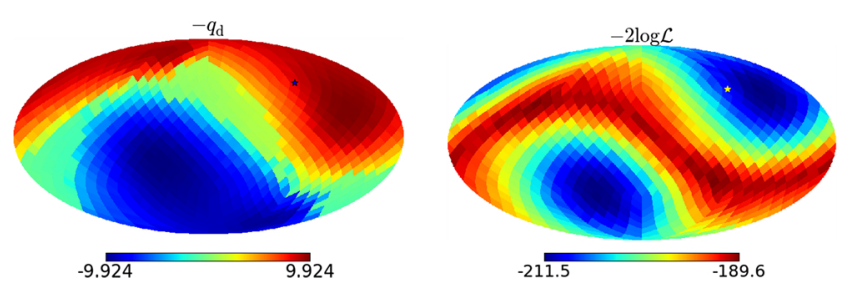

Fig. 4. Results of an a posteriori grid scan (left panel) varying the direction of the scale-dependent dipolar modulation of the form $q_{0}=$ $q_{\mathrm{m}}+\boldsymbol{q}_{\mathrm{d}} . \hat{n} \exp (-z / S)$ in galactic coordinates. The best-fit direction is within $23^{\circ}$ of the $\mathrm{CMB}$ dipole (indicated by a star) and $-2 \log \mathcal{L}$ (right panel) changes by just 3.22 between these two directions.

We also study the effect of allowing an additional uncorrelated velocity dispersion $c \sigma_{z}$ in the fit, rather than fixing it to be $150 \mathrm{~km} \mathrm{~s}^{-1}$ as in the JLA analysis (Betoule et al. 2014). As shown in Table 3 this improves the overall fit even further for $c \sigma_{z}=241 \mathrm{~km} \mathrm{~s}^{-1}$; the best-fit dipole drops a little to $q_{\mathrm{d}}=-6.33$, while the monopole is nearly unchanged at $q_{\mathrm{m}}=-0.154$. The $\Delta_{\text {BIC }}$ between the model with $q_{\mathrm{d}}=0$ and that with $q_{\mathrm{m}}=0$ is 4.91 , providing positive evidence against a universe that is accelerating isotropically. Our main result is thus robust in that the maximum likelihood estimator prefers to interpret the data as evidence of a dipole in the deceleration parameter aligned with the $\mathrm{CMB}$ dipole, rather than as an isotropic acceleration of the universe, which may indicate the presence of a cosmological constant.

As an a posteriori test, we examine the direction dependence of this scale-dependent dipolar modulation in $q_{0}$, by scanning the direction of $q_{d}$ on a grid corresponding to a HEALpix (Gorski et al. 2005) map of nside $=8$. The best-fit direction is 23 degrees away from the CMB dipole, where $q_{\mathrm{d}}$ increases to -9.851 but $-2 \log \mathcal{L}$ improves by only 3.22 . This demonstrates that the direction of the anisotropy we find is also robust. 


\section{Discussion}

It has been observed (Bernal et al. 2017) that the deceleration parameter inferred from previous $\mathrm{SNe}$ Ia datasets has a redshift, and indeed directional dependence. This was interpreted as indicative of local anisotropy in the matter distribution, i.e. our being located in an asymmetric void. The refinement in the present work is that we consider a recent comprehensive database of SNe Ia and take into account all systematic effects as encoded in the covariance matrices provided (Betoule et al. 2014). Moreover we focus on the local velocity rather than the density field as this fully reflects the gravitational dynamics due to inhomogeneities. We can then explore the expected consequences of our being tilted, i.e. non-Copernican observers. Our analysis is guided by the suggestion that we may then infer acceleration even when the overall expansion rate is decelerating - a signature of which would be a dipolar modulation of the inferred $q_{0}$ along the direction of the bulk flow.

The effect of peculiar velocities on SNe Ia cosmology has been discussed earlier (Hui \& Greene 2006; Davis et al. 2011), however these studies relied on covariances that apply to Copernican observers. As we show elsewhere (Colin et al.2019), the bulk flow we are embedded in is rare at a level of $\lesssim 1 \%$ (Rameez et al. 2018) according to the Dark Sky simulation (Skillman et al. 2014), but the conditional covariances can be up to a factor of $\sim 10$ larger and introduce a preferred direction locally. This can make a much bigger impact on cosmological inferences than was found in previous studies. In particular the JLA analysis (Betoule et al. 2014) of the same dataset claimed that the effect of peculiar velocities is a tiny $(<0.1 \%)$ shift in the best-fit cosmological parameters.

In summary, the model-independent evidence for acceleration of the Hubble expansion rate from the largest public catalogue of $\mathrm{SNe}$ Ia is only $1.4 \sigma$. This is in contrast to the claim (Scolnic et al. 2018) that acceleration is established by SNe Ia at $>6 \sigma$ in the framework of the $\Lambda \mathrm{CDM}$ model. Moreover there is a significant $(3.9 \sigma)$ indication for a dipole in $q_{0}$ towards the $\mathrm{CMB}$ dipole, which is indeed expected if the apparent acceleration is an artefact of our being located in a local bulk flow which extends out far enough to include most of the supernovae studied (Tsagas 2010, 2011; Tsagas \& Kadiltzoglou 2015). Given the observational evidence that there is no convergence to the CMB frame as far out as redshift $z \sim 0.1$, which includes half the known SNe Ia, this possibility must be taken seriously.

Acknowledgements. We thank the JLA collaboration for making all their data public and Dan Scolnic for correspondence concerning the Pantheon catalogue. We are grateful to Mike Hudson and Christos Tsagas for discussions. MR acknowledges a Carlsberg distinguished postdoctoral fellowship and hospitality at the Institut d'Astrophysique, Paris.

\section{References}

Bernal, C., Cárdenas, V. H., \& Motta, V. 2017, Phys. Lett. B, 765, 163 Betoule, M., Kessler, R., Guy, J., et al. 2014, A\&A, 568, A22

Carrick, J., Turnbull, S. J., Lavaux, G., \& Hudson, M. J. 2015, MNRAS, 450, 317

Colin, J., Mohayaee, R., Sarkar, S., \& Shafieloo, A. 2011, MNRAS, 414, 264

Colin, J., Mohayaee, R., Rameez, M., \& Sarkar, S. 2019, MNRAS, submitted

Conley, A., Guy, J., Sullivan, M., et al. 2011, ApJS, 192, 1

Davis, T. M., Hui, L., Frieman, J. A., et al. 2011, ApJ, 741, 67

Feindt, U., Kerschhaggl, M., Kowalski, M., et al. 2013, A\&A, 560, A90

Feldman, H. A., Watkins, R., \& Hudson, M. J. 2010, MNRAS, 407, 2328

Gorski, K., Hivon, E., Banday, A., et al. 2005, ApJ, 622, 759

Hellwing, W. A., Nusser, A., Feix, M., \& Bilicki, M. 2017, MNRAS, 467, 2787

Hess, S., \& Kitaura, F. S. 2014, MNRAS, 456, 4247

Hogg, D. W., Eisenstein, D. J., Blanton, M. R., et al. 2005, ApJ, 624, 54

Hudson, M. J., Smith, R. J., Lucey, J. R., \& Branchini, E. 2004, MNRAS, 352, 61

Hui, L., \& Greene, P. B. 2006, Phys. Rev. D, 73, 123526

Kashlinsky, A., Atrio-Barandela, F., Kocevski, D., \& Ebeling, H. 2009, ApJ, 686, L49

Kashlinsky, A., Atrio-Barandela, F., Ebeling, H., Edge, A., \& Kocevski, D. 2010, ApJ, 712, L81

Kashlinsky, A., Atrio-Barandela, F., \& Ebeling, H. 2011, ApJ, 732, 1

Kogut, A., Lineweaver, C., Smoot, G. F., et al. 1993, ApJ, 419, 1

Lauer, T. R., \& Postman, M. 1994, ApJ, 425, 418

Lavaux, G., Tully, R. B., Mohayaee, R., \& Colombi, S. 2010, ApJ, 709, 483

Magoulas, C., Springob, C., Colless, M., et al. 2016, Proc. IAU Symp., 308, 336

March, M., Trotta, R., Berkes, P., Starkman, G., \& Vaudrevange, P. 2011, MNRAS, 418, 2308

Neill, J. D., Hudson, M. J., \& Conley, A. 2007, ApJ, 661, L123

Nielsen, J. T., Guffanti, A., \& Sarkar, S. 2016, Sci. Rep., 6, 35596

Peebles, P.J.E. 1994, Principles of Physical Cosmology (Princeton University Press)

Perlmutter, S., Aldering, G., Goldhaber, G., et al. 1999, ApJ, 517, 565

Planck Collaboration I. 2019, A\&A, in press, https://doi.org/10.1051/ Q004-6361/201833880

Rameez, M. 2019, ArXiv e-prints [arXiv:1905.00221]

Rameez, M., Mohayaee, R., Sarkar, S., \& Colin, J. 2018, MNRAS, 477, 1772

Riess, A. G., Filippenko, A. V., Challis, P., et al. 1998, AJ, 116, 1009

Riess, A. G., Strolger, L. G., Casertano, S., et al. 2007, ApJ, 659, 98

Rubin, D., \& Hayden, B. 2016, ApJ, 833, L30

Scolnic, D. M., Jones, D. O., Rest, A., et al. 2018, ApJ, 859, 101

Scrimgeour, M. I., Davis, T., Blake, C., et al. 2012, MNRAS, 425, 116

Shariff, H., Jiao, X., Trotta, R., \& van Dyk, D. A. 2016, ApJ, 827, 1

Skillman, S.W., Warren, M.S., \& Turk, M.J. 2014, ArXiv e-prints [arXiv:1407.2600]

Schwarz, G. 1978, Ann. Stat., 6, 461

Schwarz, D. J., Copi, C. J., Huterer, D., \& Starkman, G. D. 2016, Class. Quant. Grav., 33, 184001

Tsagas, C. 2010, MNRAS, 405, 503

Tsagas, C. 2011, Phys. Rev. D, 84, 063503

Tsagas, C., \& Kadiltzoglou, M. I. 2015, Phys. Rev. D, 92, 043515

Tutusaus, I., Lamine, B., Dupays, A., \& Blanchard, A. 2017, A\&A, 602, A73

Visser, M. 2004, Class. Quant. Grav., 21, 2603

Watkins, R., Feldman, H. A., \& Hudson, M. J. 2009, MNRAS, 392, 743

Watkins, R., \& Feldman, H. A. 2015, MNRAS, 447, 132 


\section{Appendix A: Redshift-dependence of light-curve fitting parameters}

In this work we have used a statistical approach as well as the treatment of light-curve parameters espoused by Nielsen et al. (2016). These authors were criticised by Rubin \& Hayden (2016) for using redshift-independent distributions for $x_{1}$ and $c$. In this respect we note the following:

1. The JLA analysis determined the relationship between the luminosity distance and redshift for $\mathrm{SNe}$ Ia. To inspect a posteriori the distribution of two $\left(x_{1}\right.$ and $\left.c\right)$ out of the three ingredients that go into standardising SNe Ia and then add empirical terms in the fit to describe their sample dependence and redshift evolution, is fundamentally against the principles of blind hypothesis testing, especially since no such dependence had been suggested by Betoule et al. (2014).

2. Nevertheless we carry out the same 22-parameter fit (Rubin \& Hayden 2016) for comparison and present the results in Table A.1. While the log maximum likelihood ratio does improve for these fits, this parameterisation increases the significance of the dipole in $q_{0}$ to $4.7 \sigma$ (likelihood ratio of 18.3) and reduces further the significance of a monopole.

3. The addition of parameters to improve the quality of a fit and obtain a desired outcome have to be justified by physical and/or information theoretic arguments. The additional parameters of Rubin \& Hayden (2016) can be justified by the Akaike information criterion but not by the stricter Bayesian information criterion. This also applies to the two additional parameters we introduce $\left(q_{\mathrm{d}}\right.$ and $\left.S\right)$ but these are physically motivated for a tilted observer (Tsagas 2011; Tsagas \& Kadiltzoglou 2015).

4. If the light-curve parameters $x_{1}$ and $c$ are allowed to be sample- or redshift-dependent we can ask why the absolute magnitude of SNe Ia should also not be sample- or redshiftdependent. Allowing this of course undermines their use as standard candles and the data is then unsurprisingly consistent with no acceleration (Tutusaus et al. 2017), as seen in Table A.1.

\section{Appendix B: Uncertainties}

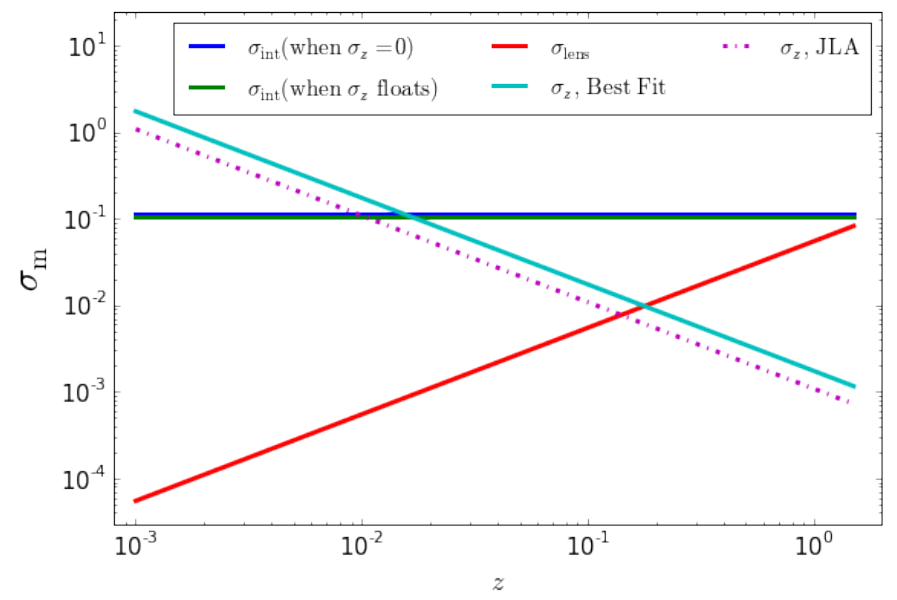

Fig. B.1. Different sources of intrinsic dispersion in magnitude $\sigma_{\mathrm{m}}$ that enter cosmological fits of JLA data. On top of the global $\sigma_{\text {int }}$, SNe Ia are given a dispersion $\sigma_{\text {lens }}$ proportional to redshift to account for lensing, while low redshift SNe Ia are selectively more dispersed by $\sigma_{z}$ to account for peculiar velocity effects.

The JLA covariance matrix includes uncertainties from, for example the light-curve template fitting process, calibration uncertainties, and dust extinction in the Galaxy, together with the expected dispersion resulting from peculiar velocities (which mainly affects low redshift $\mathrm{SNe}$ ) and lensing (which mainly affects high redshift $\mathrm{SNe}$ Ia) and the propagated uncertainties from the flow model from which the SN by SN peculiar velocity corrections are performed. In addition it is also necessary to fit for a global intrinsic dispersion as in previous analyses (March et al. 2011). We use heliocentric redshifts in this analysis and thus do not include uncertainties related to the peculiar velocity corrections based on the flow model. The redshift dependence of the dispersions in the fit are shown in Fig. B.1.

Table A.1. Fits to the JLA catalogue allowing for sample- and redshift-dependence of SNe Ia parameters.

\begin{tabular}{|c|c|c|c|c|c|c|c|c|c|}
\hline & $-2 \log \mathcal{L}_{\max }$ & $q_{\mathrm{m}}$ & $q_{\mathrm{d}}$ & $S$ & $j_{0}-\Omega_{k}$ & $\alpha$ & $\beta$ & $M_{0}$ & $\sigma_{M_{0}}$ \\
\hline $\begin{array}{l}\mathrm{R} \& \mathrm{H}(22 \text { parameters }) \text { with peculiar velocity } \\
\text { corrections and no dipole }\left(q_{\mathrm{d}}=0\right)\end{array}$ & -331.6 & -0.457 & 0 & - & 0.146 & 0.135 & 3.07 & -19.074 & 0.107 \\
\hline - as above, with no acceleration $\left(q_{\mathrm{m}}=0\right)$ & -315.6 & 0 & 0 & - & -1.35 & 0.132 & 3.05 & -19.013 & 0.109 \\
\hline $\begin{array}{l}\mathrm{R} \& \mathrm{H}(22 \text { parameters }) \text { with no peculiar velocity } \\
\text { corrections and no dipole }\left(q_{\mathrm{d}}=0\right)\end{array}$ & -306.70 & -0.333 & 0 & - & -0.397 & 0.133 & 3.00 & -19.050 & 0.116 \\
\hline - as above, with no acceleration $\left(q_{\mathrm{m}}=0\right)$ & -298.15 & 0 & 0 & - & -1.37 & 0.132 & 2.98 & -19.011 & 0.117 \\
\hline $\begin{array}{l}\mathrm{R} \& \mathrm{H}(24 \text { parameters }) \text { with no peculiar velocity } \\
\text { corrections and dipole } \propto \mathrm{e}^{-z / S}\end{array}$ & -325.00 & -0.310 & -8.09 & 0.0256 & -0.471 & 0.135 & 3.01 & -19.053 & 0.113 \\
\hline - as above with no dipole $\left(q_{\mathrm{d}}=0\right)$ & -306.70 & -0.333 & 0 & 0 & -0.400 & 0.134 & 3.00 & -19.054 & 0.116 \\
\hline - as above, with no acceleration $\left(q_{\mathrm{m}}=0\right)$ & -318.14 & 0 & -6.19 & 0.0344 & -1.32 & 0.133 & 3.00 & -19.012 & 0.114 \\
\hline
\end{tabular}

Notes. The first row corresponds to the 22-parameter fit of Rubin \& Hayden (2016) with the full JLA covariances. In the second row, the peculiar velocity corrections they made are undone (and the corresponding arbitrary uncertainties imposed are excluded). The third row demonstrates the dramatic improvement in the fit when a scale-dependent exponentially falling dipole (with 2 additional parameters) is allowed for in $q_{0}$. 